\title{
Inhibition of Akt sensitises neuroblastoma cells to gold(III) porphyrin la, a novel antitumour drug induced apoptosis and growth inhibition
}

\author{
W Li ${ }^{1,2}$, Y Xie', RW-Y Sun ${ }^{3}$, Q Liu', J Young', W-Y Yu', C-M Che ${ }^{*, 3}$, PK Tam ${ }^{*, 1}$ and Y Ren ${ }^{*, 1,4}$ \\ 'Department of Surgery, University of Hong Kong, Pokfulam Road, Hong Kong, PR China; ${ }^{2}$ Laboratory in Department of Surgery, The First Affiliated \\ Hospital, Guangzhou Medical College, Guangzhou, Guangdong, PR China; ${ }^{3}$ Department of Chemistry, University of Hong Kong, Pokfulam Road, Hong \\ Kong, PR China; ${ }^{4}$ Department of Cell Biology and Neuroscience, Rutgers University, NJ 08854, USA
}

BACKGROUND: Gold(III) porphyrin Ia is a new class of anticancer drug, which inhibits cell proliferation of wide range of human cancer cell lines and induces apoptosis in human nasopharyngeal carcinoma cells. However, the underlying signalling mechanism by which gold(III) porphyrin I a modifies the intracellular apoptosis pathways in tumour cells has not been explained in detail in neuroblastoma cells.

METHODS: Cell proliferation and apoptosis were determined by measuring 3-(4,5-dimethylthiazol-2-yl)-2,5-diphenyltetrazolium bromide (MTT) and Annexin $\vee$ binding, respectively. Western blot assay was used to detect proteins involved in apoptotic and Akt pathways. In vivo tumour growth was assessed by inoculating tumour cells to nude mice subcutaneously, and gold(III) porphyrin Ia was administrated intravenously.

RESULTS: This study assessed the antitumour effect and mechanism of gold(III) porphyrin Ia on neuroblastoma in vitro and in vivo. Gold(III) porphyrin I a displayed a growth inhibition and induction of apoptosis in neuroblastoma cells effectively in vitro, which was accompanied with release of cytochrome $c$ and Smac/DIABLO and caspases activation. Further studies indicated that gold(III) porphyrin I a inhibited X-linked inhibitor of apoptosis (XIAP). However, we found that gold(III) porphyrin Ia can induce a survival signal, Akt activation within minutes and could last for at least $24 \mathrm{~h}$. To further confirm association between activation of Akt and the effectiveness of gold(III) porphyrin Ia, neuroblastoma cells were treated with API-2, an Akt-specific inhibitor. API-2 sensitised cells to gold(III) porphyrin la-induced apoptosis and growth inhibition.

CONCLUSION: These results suggested that Akt may be considered as a molecular 'brake' that neuroblastoma cells rely on to slow down gold(III) porphyrin Ia-induced apoptosis and antiproliferation. Gold(III) porphyrin Ia is a mitochondrial apoptotic stimulus but also activates Akt, suggesting an involvement of Akt in mediating the effectiveness to growth inhibition and apoptosis by gold(III) porphyrin Ia and that inhibition of Akt can enhance the anticancer activity of gold(III) porphyrin Ia in neuroblastoma. British Journal of Cancer (2009) I 0I, 342-349. doi:I0.1038/sj.bjc.6605 I47 www.bjcancer.com

Published online 23 June 2009

(c) 2009 Cancer Research UK

Keywords: Akt; apoptosis; caspases; gold(III) porphyrin Ia; neuroblastoma

Neuroblastoma is one of the most common malignant tumours in children, and is responsible for about $15 \%$ of all paediatric cancer deaths. (Maris and Matthay, 1999) Spontaneous regressions and differentiation in vivo are common in infants and in early-stage

*Correspondence: Dr Y Ren, Department of Cell Biology and Neuroscience, Rutgers University, 604 Allison Road, D-25I, Piscataway, NJ 08854, USA; E-mail: ren@dls.rutgers.edu

or Professor C-M Che, Department of Chemistry and Open Laboratory of Chemical Biology of the Institute of Molecular Technology for Drug Discovery and Synthesis, University of Hong Kong, Pokfulam Road, Hong Kong, PR China; E-mail: cmche@hku.hk

or Professor PK Tam, Department of Surgery, University of Hong Kong, Pokfulam Road, Hong Kong, PR China; E-mail: paultam@hkucc.hku.hk Received 22 December 2008; revised 7 May 2009; accepted 28 May 2009; published online 23 June 2009 tumours, whereas children who are over 1 year of age at clinical presentation often have metastatic disease that fails to respond to current therapies, including intensive chemotherapy, irradiation therapy, and surgery. (Matthay et al, 1999; Weinstein et al, 2003) The underlying biological mechanism has not been fully elucidated. Despite recent advances in the management of the disease by conventional chemotherapy, the development of new therapeutic strategies is critically important.

Apoptosis is mediated by two central pathways: the extrinsic (or death receptor) and the intrinsic (or mitochondrial) pathways (Danial and Korsmeyer, 2004). The intrinsic pathway is initiated through either the disruption of the mitochondrial membrane potential directly or indirectly through upstream effector molecules. This disruption leads to the release of cytochrome $c$ and the formation of the apoptosome complex of cytochrome $\mathrm{c} /$ caspase 9/ Apaf- 1 and activation of the caspase cascade leading to apoptosis. Several endogenous proteins antagonize the intrinsic pathway such 
as X-linked inhibitor of apoptosis (XIAP). X-linked inhibitor of apoptosis binds to and inhibits caspase-9, 3, and 7 (Deveraux et al, 1998). The release of Smac/DIABLO from mitochondrial to the cytosol opposes the inhibition of caspases by XIAP (Du et al, 2000). This relief of inhibition is mediated by binding Smac/ DIABLO to XIAP, displacing the caspases. The role of Smac/ DIABLO emphasizes that although release of cytochrome $c$ is necessary, it is not sufficient in many situations to bring about cell death (Potts et al, 2003). Inactivation of the 'cell death brakes' by these other apoptogens is also often necessary for apoptosis to occur (Crow et al, 2004).

The phosphatidylinositol 3 '-kinase (PI3K) - Akt signalling pathway regulates fundamental cellular functions such as transcription, translation, proliferation, growth, and survival (Osaki et al, 2004). It has been shown that Akt is capable of interfering with both the intrinsic (mitochondrial) and extrinsic (receptor mediated) apoptotic pathways (Nicholson and Anderson, 2002). Inhibiting Akt signalling in tumour cells led to selective induction of apoptosis in tumour cells expressing activated Akt. In contrast, a minimal effect was observed in normal or tumour cells expressing low levels of active Akt (Jetzt et al, 2003). These studies state the importance of Akt activation in tumour cell survival. Recent studies have shown that the activation of Akt also contributes to both chemotherapeutic resistance and radiation resistance in nonsmall cell lung cancer and other carcinoma (Brognard et al, 2001; Clark et al, 2002). However, the importance of Akt in promoting therapeutic resistance in neuroblastoma cells has not been established.

Gold(III) porphyrin 1a is a novel antitumor chemotherapeutic reagent (Che et al, 2003). Preliminary data showed that gold(III) porphyrin 1a inhibited cell proliferation of wide range of human cancer cell lines, including human cervical epithelioid cancer cells, leukaemia, hepatocellular, and nasopharyngeal carcinoma cells (Che et al, 2003; Wang et al, 2005) in vitro, suggesting that this molecule may offer novel approach to cancer therapy. Very recent data showed that gold(III) porphyrin 1a induced apoptosis in human nasopharyngeal carcinoma cells through mitochondrial death pathways related to reactive oxygen species (ROS) (Wang et al, 2005, 2006). However, the underlying signalling mechanism by which gold(III) porphyrin 1a modifies the intracellular apoptosis pathways in tumour cells has not been explained in detail in neuroblastoma cells. Thus, in this study, we reported that gold(III) porphyrin 1a induced neuroblastoma cell apoptosis and growth inhibition both in vitro and in vivo. We showed that gold(III) porphyrin 1a-induced apoptosis was through the activation of caspases and mitochondria pathway. Most interestingly, gold(III) porphyrin 1a lead to the activation of Akt before the onset of apoptosis, suggesting that early activation of Akt by gold(III) porphyrin 1a may act as a molecular brake on gold(III) porphyrin 1a-induced apoptosis and thus may serve as new mechanism of therapeutic resistance. Inhibition of Akt activity might increase therapeutic efficacy of gold(III) porphyrin 1a in neuroblastoma.

\section{MATERIALS AND METHODS}

\section{Reagents}

Gold(III) porphyrin 1a was synthesised and purified by a method described previously (Che et al, 2003). Antibodies for phosphorylated Akt (p-Akt), Akt, caspase-3, caspase-9, PARP, and $\beta$-actin were from Cell Signaling (Cell Signaling Technology, Beverly, MA, USA). Chemiluminescence Western Blot detection reagents were from Perkin-Elmer (Boston, MA, USA). All other chemicals were purchased from Sigma (St Louis, MO, USA). Tissue culture plastic was purchased from Corning (Corning, NY, USA). Cell culture medium DMEM, fetal bovine serum (FBS) and antibiotics were from Mediatech Inc. (Herndon, VA, USA).

\section{Cell lines and culture}

Neuroblastoma cell lines SK-N-AS and SK-N-SH were obtained commercially from American Type Culture Collection (ATCC, Rockville, MD, USA) and cells were cultured with DMEM medium supplemented with $10 \%(\mathrm{v} / \mathrm{v})$ heat inactive $\mathrm{FBS}, 100 \mathrm{U} \mathrm{ml}^{-1}$ penicillin and $100 \mu \mathrm{g} \mathrm{ml}^{-1}$ streptomycin in a humidified $5 \% \mathrm{CO}_{2}$ incubator at $37^{\circ} \mathrm{C}$. The human non-tumorigenic, immortalised liver cell line MIHA was maintained in Waymouth's MB 752/1 medium (Gibco BRL, Grand Island, NY, USA) supplemented with $10 \% \mathrm{FBS}, 100 \mathrm{U} \mathrm{ml}^{-1}$ penicillin, $100 \mathrm{mg} \mathrm{ml}^{-1}$ streptomycin, $50 \mathrm{~mm}$ dexamethasone and $20 \mathrm{mU} \mathrm{ml}^{-1}$ insulin (Boehringer Mannheim, Indianapolis, IN, USA).

\section{Apoptosis assay}

Apoptosis was detected by fluorescein-labelled Annexin V staining using the Annexin V-Fluos staining kit (Roche, Mannheim, Germany). Cells were treated with gold(III) porphyrin 1a at different concentrations. Cells were washed with ice-cold phosphate-buffered saline (PBS), trypsinised, and labelled with Annexin $\mathrm{V}$ for $15 \mathrm{~min}$ at room temperature in the dark. The percentage of FITC-positive cells was analysed by flow cytometry (FACScaliber, Becton Dicknison, Mountain View, CA, USA). The data were analysed using CellQuest software (Becton Dicknison).

\section{Proliferation assay}

Cells were treated with different concentrations of gold(III) porphyrin la from 0.125 to $1.0 \mu \mathrm{m}$ for $48 \mathrm{~h}$. Cell proliferation was determined by measuring 3-(4,5-dimethylthiazol-2-yl)-2,5-diphenyltetrazolium bromide (MTT) reduction following the protocol (Cell Proliferation kit, BD Biosciences, San Jose, CA, USA), with a microtiter plate reader (Bio-Rad Model 550, Hercules, CA, USA) at $570 \mathrm{~nm}$. Optical density was measured at $570 \mathrm{~nm}$. Data were obtained from three separate experiments. The percentage of cell survival was determined by comparing the absorbance value of the vehicle control.

\section{Isolation of cytosolic fractions}

The assay was performed according to the method of (Bossy-Wetzel et al (1998) with minor modifications. Cells were washed in cold PBS twice and resuspended in mitochondria isolation buffer $(20 \mathrm{~mm}$ Hepes, pH 7.5, $1.5 \mathrm{~mm} \mathrm{MgCl} 2,10 \mathrm{~mm} \mathrm{KCl,} 1$ mm EDTA, 1 mM EGTA, $1 \mathrm{~mm}$ dithiothreitol, $0.1 \mathrm{~mm}$ phenylmethylsulfonyl fluoride, $10 \mu \mathrm{g} \mathrm{ml}^{-1}$ leupeptin, aprotinin, and pepstatin containing $250 \mathrm{~mm}$ sucrose). Cells were homogenised in an ice-cold tissue grinder with 10 strokes. The homogenate was centrifuged at $900 \mathrm{~g}$ for $10 \mathrm{~min}$. The resulting supernatant was centrifuged at $13000 \mathrm{~g}$ for $15 \mathrm{~min}$ and pellet was designated as mitochondria. The supernatant was further centrifuged at $13000 \mathrm{~g}$ to remove any other particulate material and supernatant was used as the cytosolic fraction.

\section{Western blot}

Cells were washed with PBS, and directly lysed in lysis buffer (50 mM Tris- $\mathrm{HCl}(\mathrm{pH}=8.0), 1 \%$ Triton $\mathrm{X}-100,10 \%$ glycerol, $1 \mathrm{~mm}$ EDTA, $250 \mathrm{~mm} \mathrm{NaCl}, 1 \mathrm{~mm}$ dithiothreitol, $1 \mathrm{~mm}$ phenylmethylsulfonyl fluoride, $2 \mathrm{~mm}$ sodium vanadate, $100 \mathrm{~mm}$ sodium fluoride, $10 \mu \mathrm{g} \mathrm{ml}^{-1}$ aprotinin, $10 \mu \mathrm{g} \mathrm{ml}^{-1}$ leupeptin and $10 \mu \mathrm{g} \mathrm{ml}^{-1}$ pepstatin). Cell lysates were adjusted to equal protein concentrations (Bio-Rad Protein Assay, Hercules, CA, USA), resuspended in $2 \times$ sample loading buffer containing $4 \%$ SDS, $20 \%$ glycerol, $120 \mathrm{~mm}$ Tris and bromophenol blue, and were boiled for $5 \mathrm{~min}$. Protein samples were subjected to SDS-polyacrylamide gel electrophoresis. Proteins on the gel were transferred onto nitrocellulose membranes that were blocked with $5 \%$ milk in Tris-buffered saline containing $0.1 \%$ 

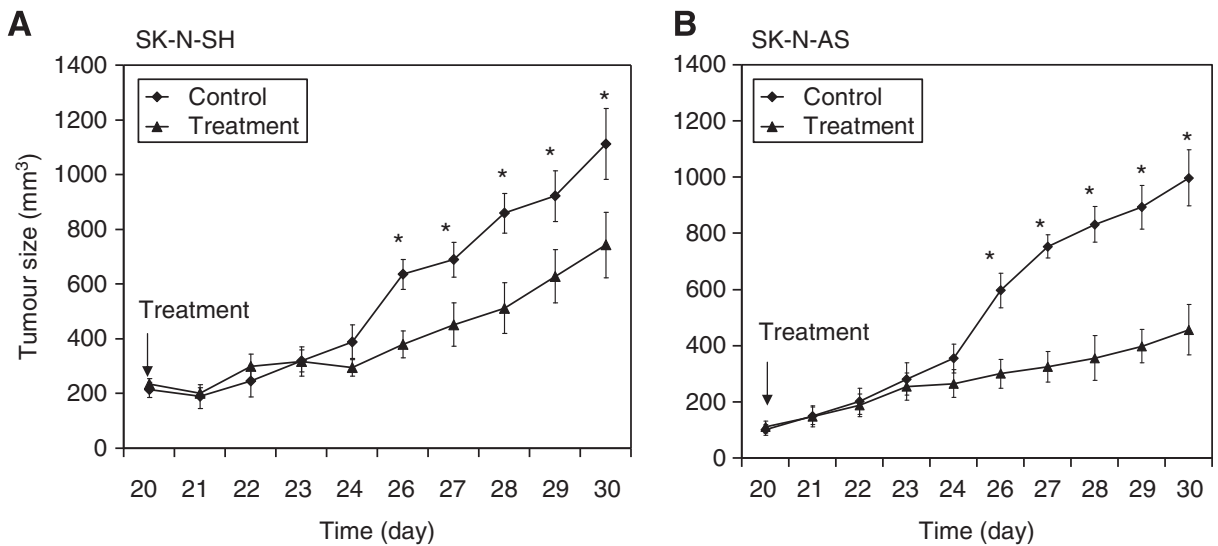

Figure I Antitumor effect of gold(III) porphyrin Ia in BALB/c-nude mice. Mice were inoculated with SK-N-SH (A) and SK-N-AS (B) subcutaneously with $1 \times 10^{6}$ cells. Tumours developed 20 days after injection and mice were administered gold(III) porphyrin la every 3 days intravenously until day 2 | postinoculation. Control group received vehicle alone ( $10 \%$ ethanol). The size of tumours in mice was monitored everyday. Results are expressed as the mean $(n=10$ per group, $* P<0.05)$.

Tween 20 (TBST) for $1 \mathrm{~h}$ at room temperature. Afterwards, the membranes were incubated with the indicated primary antibodies overnight at $4^{\circ} \mathrm{C}$. After being washed with TBST, the membranes were incubated with the appropriate secondary antibody. The immunoreactive bands were visualised with chemiluminescence.

\section{Tumour growth in nude mice}

Six-week-old BALB/c nude mice (Laboratory Animal Unit, The University of Hong Kong) were injected with neuroblastoma cell lines SK-N-SH and SK-N-AS subcutaneously with $1 \times 10^{6}$ cell. After 20 days, all of the nude mice developed the tumours. For treatment group $(n=10), 10 \mathrm{mg} \mathrm{kg}^{-1}$ gold(III) porphyrin 1a dissolved in $10 \%$ ethanol was administrated intravenously on day 21 postinoculation. Control group $(n=10)$ received $10 \%$ ethanol vehicle. Tumour growth in each group was monitored everyday by two-dimensional measurements of individual tumours.

\section{RESULTS}

\section{Effects of gold(III) porphyrin la on tumour growth in vivo}

Mice were inoculated with neuroblastoma cell lines SK-N-AS and SK-N-SH subcutaneously with $1 \times 10^{6}$ cells. SK-N-SH cell line possesses multidrug resistance (MDR) phenotype and SK-N-AS cell line is non-MDR phenotype. (Cinatl et al, 1999) Mice were administered gold(III) porphyrin $1 \mathrm{a}\left(10.0 \mathrm{mg} \mathrm{kg}^{-1}\right)$ every 3 days intravenously till day 21 postinoculation. Control group received vehicle alone. Tumour growth in each group was monitored everyday by two-dimensional measurements of individual tumours. In control group, tumours in mice inoculated with SK-N-AS and SK-N-SH grew very progressively. However, treatment with gold(III) porphyrin 1a resulted in strong and significant decreases in tumour size both in mice inoculated with SK-N-AS and SK-N-SH (Figure 1A and B), suggesting that gold(III) porphyrin 1a not only inhibited non-MDR neuroblastoma growth (SK-N-AS) but also was effective for MDR phenotype neuroblastoma (SK-N-SH). In addition, non-MDR cell line SK-N-AS responded significantly better than the MDR cell line SK-N-SH $(P<0.05)$.

\section{Antiproliferative effects and apoptosis induction of gold (III) porphyrin 1a}

Given these findings in vivo, we further analysed how gold(III) porphyrin 1a affects tumour growth in vitro. The effects of gold(III) porphyrin 1a on cell proliferation were assessed in the human neuroblastoma cell lines SK-N-SH and SK-N-AS. Gold(III) porphyrin 1a-induced growth inhibitory effects occurred in SK-NSH and SK-N-AS with $\mathrm{IC}_{50}$ values 0.2 and $0.4 \mu \mathrm{M}$, respectively. However, under the same conditions, the effect of gold(III) porphyrin 1a on normal live cell (MIHA) was lowered when compared with SK-N-SH and SK-N-AS (Figure 2A). SK-N-SH cell line has MDR phenotype and the $\mathrm{IC}_{50}$ values for cisplatin, a common first-line chemotherapeutic drug in clinical setting, exceeded $40 \mu \mathrm{m}$ for SK-N-SH and was $10.5 \mu \mathrm{M}$ for SK-N-AS (Table 1). SK-N-SH is resistant to cisplatin treatment; however, the sensitivity to gold(III) porphyrin 1a is 100 times more than cisplatin.

To understand whether gold(III) porphyrin 1a has the effect on tumour cell apoptosis, cells were treated with gold(III) porphyrin 1a for 12 or $24 \mathrm{~h}$ and apoptosis was assessed by Annexin $\mathrm{V}$ binding assay. Gold(III) porphyrin 1a induced apoptosis in SK-N-AS and SK-N-SH but had no effect in normal live cells. Similarly, gold(III) porphyrin 1a induced more apoptosis at $24 \mathrm{~h}$ compared with $12 \mathrm{~h}$ (Figure 2B and C). Therefore, gold(III) porphyrin 1a-elicited neuroblastoma cell apoptosis was in time- and dose-dependent manners.

\section{Gold(III) porphyrin 1a induced release of cytochrome $c$ and Smac/Diablo}

We further explored the mechanism of gold(III) porphyrin 1ainduced apoptosis of neuroblastoma cells. Apoptotic stimuli can damage mitochondrial, resulting in cytochrome $c$ and mitochondrial protein Smac/Diablo release into the cytosol. Having previously reported the release of cytochrome $c$ with gold(III) porphyrin 1a treatment in human nasopharyngeal carcinoma cells (Wang et al, 2005), we next assessed whether Smac/Diablo was also released. SK-N-SH and SK-N-AS were treated with gold(III) porphyrin 1a $(0.5 \mu \mathrm{M})$ for $8 \mathrm{~h}$ and cytosols were separated. As showed in Figure 3A, Smac/Diablo was released to cytosol in a pattern similar to cytochrome $c$.

\section{Gold(III) porphyrin 1a treatment resulted in downregulation and cleavage of XIAP}

Smac/Diablo is known to exert its proapoptotic effect by inhibition of XIAP. X-linked inhibitor of apoptosis is a member of intracellular antiapoptotic proteins that confers protection form death-inducing stimuli by directly blocking the activation of caspases (Deveraux et al, 1999). To determine the effect of XIAP in gold(III) porphyrin 1a-induced apoptotic execution, cells were treated with gold(III) porphyrin $1 \mathrm{a}(0.5 \mu \mathrm{M})$ for different periods of 

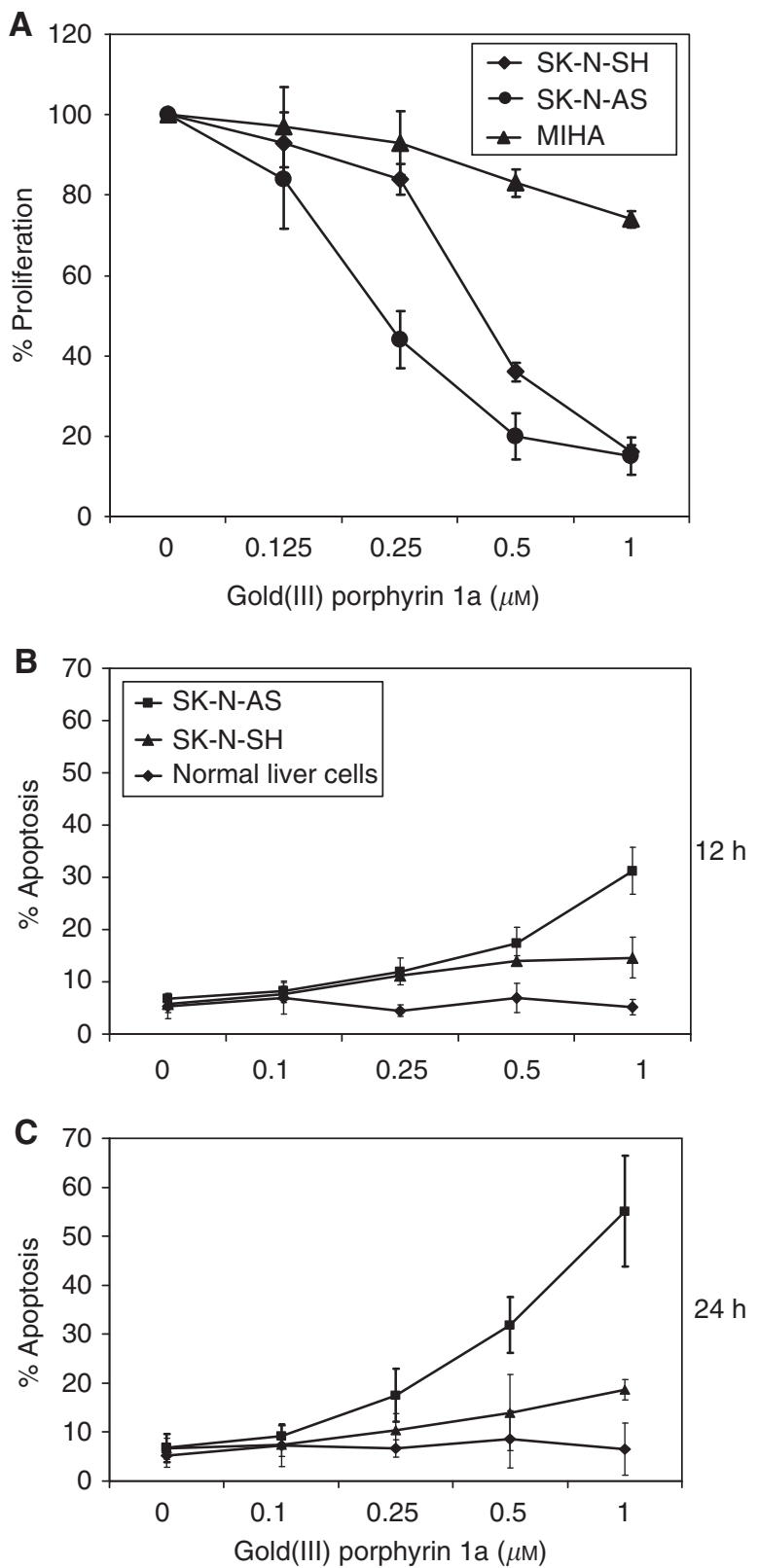

Figure 2 Proliferative and apoptotic effects of gold(III) porphyrin Ia on neuroblastoma cells. (A) SK-N-SH, SK-N-AS and MIHA (normal liver cell line) cells were cultured with gold(III) porphyrin I a for $48 \mathrm{~h}$ in 96 -well plate. The proliferation was evaluated by MTT assay. Each treatment group contained eight replicates. Data expressed as mean \pm s.d. and similar results were obtained from independent experiments. (B and C) SK-N-SH, SK-NAS, and MIHA cells were cultured with gold(III) porphyrin la for $12 \mathrm{~h}$ (B) and $24 \mathrm{~h}(\mathbf{C})$. Apoptosis of gold(III) porphyrin la on neuroblastoma cells was evaluated by Annexin $\vee$ staining. Data expressed as mean \pm s.d. and similar results were obtained from independent experiments.

time. Expression of full-length XIAP ( $53 \mathrm{kDa})$ in both SK-N-SH and SK-N-AS was markedly inhibited by gold(III) porphyrin 1a at time $24 \mathrm{~h}$ (Figure 3B). The data also showed that the time of Smac/ Diablo release preceded that of XIAP cleavage (Figure $3 \mathrm{~A}$ and $\mathrm{B}$ ).

\section{Effect of gold(III) porphyrin 1a on the activation of caspases}

Activation of caspases during apoptosis results in the cleavage and activation/inactivation of a range of critical cellular substrates,
Table I Cytotoxicity (IC 50 ) of Gold(III) porphyrin on neuroblastoma cell lines

\begin{tabular}{lcr} 
& \multicolumn{2}{c}{$\mathbf{I C}_{\mathbf{5 0}}(\boldsymbol{\mu} \mathbf{M})$} \\
\cline { 2 - 3 } Cell lines & $\mathbf{G o l d ( I I I )}$ porphyrin Ia & Cisplatin \\
\hline SK-N-AS (non-MDR phenotype) & 0.2 & 10.5 \\
SK-N-SH (MDR phenotype) & 0.4 & $>40.0$ \\
Normal liver cells & 1.75 & 38.5 \\
\hline
\end{tabular}

$\mathrm{MDR}=$ multidrug resistance

including the DNA repair enzyme PARP. To determine which caspases were involved in gold(III) porphyrin la-induced apoptosis, the expressions of activated caspases were detected by western blotting. As shown in Figure $3 \mathrm{C}-\mathrm{E}$, in control cells, caspase- 9 and -3 and PARP were all present as uncleaved forms. After the treatment with gold(III) porphyrin 1a for $24 \mathrm{~h}$, the cleavage of caspases and PARP to catalytically active fragments was clearly detected, suggesting the activation of these caspases.

\section{Gold(III) porphyrin 1a induced phosphorylation of Akt}

As Akt activation has been implicated in the promotion of tumour cell proliferation and some antitumour drugs can inhibit tumour growth by inactivating Akt, we next examined whether gold(III) porphyrin la could affect Akt. SK-N-SH and SK-N-AS cells were treated with gold(III) porphyrin $1 \mathrm{a}(0.5 \mu \mathrm{M})$ for different periods of time. Interestingly, we found that Akt activation was not inhibited by gold(III) porphyrin 1a. By contrast, gold(III) porphyrin 1a treatment resulted in Akt activation by phosphorylation of Akt at Ser473 beginning by $30 \mathrm{~min}$ in both neuroblastoma cells SK-N-AS and SK-N-SH (Figure 4), suggesting an early induction of Akt before the onset of apoptosis. Furthermore, the activation of Akt can last for at least $24 \mathrm{~h}$.

Effect of inhibition of Akt activity on the sensitivity of cells to gold(III) porphyrin 1a treatment

Because activation of Akt can protect cells from apoptosis and our data showed gold(III) porphyrin 1a-induced early activation of Akt, we therefore reasoned that whether inhibition of Akt activation might sensitise cells to gold(III) porphyrin-induced apoptosis. Akt/protein kinase B signalling inhibitor (API-2), a cell-permeable tricyclic nucleoside, selectively inhibited cellular phosphorylation of Akt but not activation of PI3K, PDK1, PKC, PKA, ERK1/2, or c-Jun NH2-terminal kinase (Yang et al, 2004). Neuroblastoma cells SK-N-AS and SK-N-SH were pretreated with or without API-2 $(1.0 \mu \mathrm{M})$ for $2 \mathrm{~h}$ and then treated with gold(III) porphyrin 1a for $24 \mathrm{~h}$. API-2 at $1.0 \mu \mathrm{M}$ can inhibit Akt activation (data not shown) but did not affect cell growth and apoptosis (Figure 5). Treatment with API-2 enhanced tumour cell sensitivity to gold(III) porphyrin 1a-induced growth inhibition in a dosedependent manner (Figure $5 \mathrm{~A}$ ). The $\mathrm{IC}_{50}$ values for gold(III) porphyrin 1a in SK-N-AS and SK-N-SH treated with API-2 were 0.1 and $0.2 \mu \mathrm{M}$ compared with 0.2 and $0.4 \mu \mathrm{M}$ in cells treated with gold(III) porphyrin 1a alone (Figure 5A). To assess how inhibition of Akt activation would affect gold(III) porphyrin 1a-induced apoptosis, cells were treated with gold(III) porphyrin alone or the combinations of API-2 $(1.0 \mu \mathrm{M})$ and gold(III) porphyrin 1a for $24 \mathrm{~h}$. When treatment was combined with API-2, gold(III) porphyrin 1a resulted in dose-dependent apoptosis induction that was greater than that observed at the same concentration of gold(III) porphyrin 1a without API-2 treatment (Figure 5B), which is consistent with the antiproliferative assay results. 
A Gold(III) Porphyrin 1a
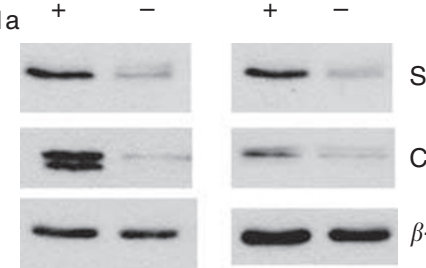

Smac

Cytochrome $c$ SK-N-SH SK-N-AS

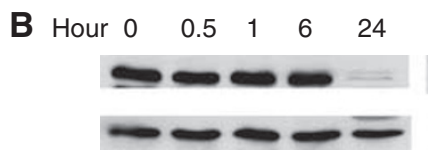

SK-N-SH

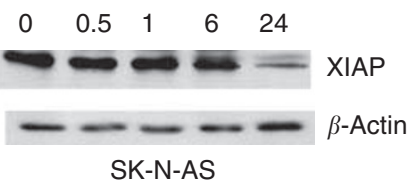

C SK-N-SH

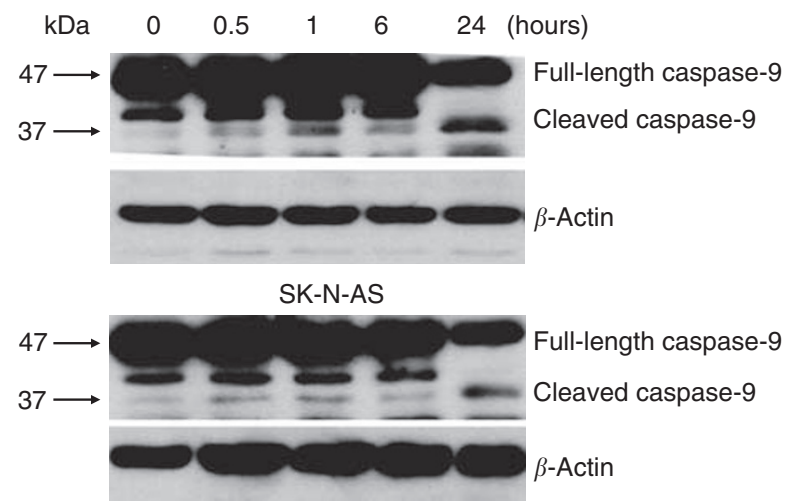

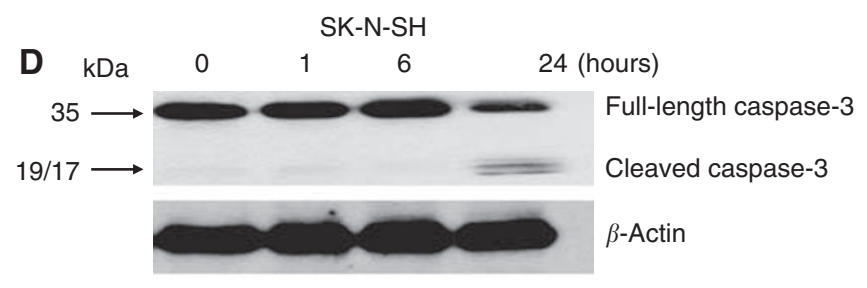

SK-N-AS

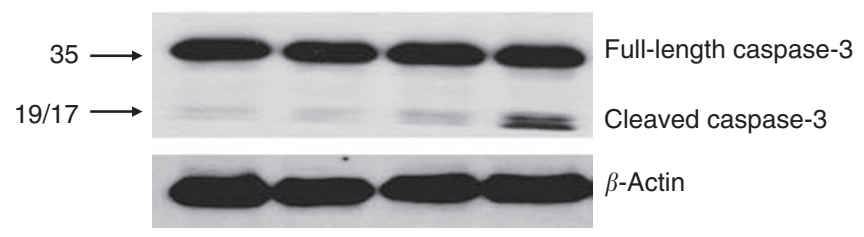

E

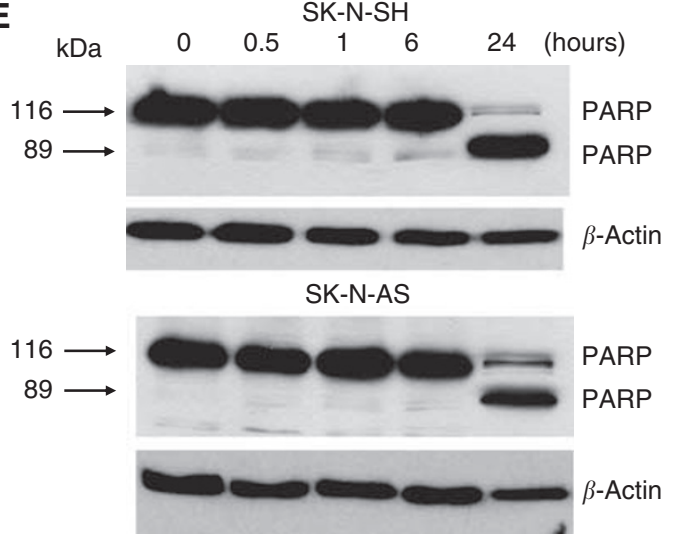

Figure 3 Characterisation of gold(III) porphyrin Ia-mediated apoptosis in neuroblastoma cells. (A) Smac and cytochrome c release in neuroblastoma cells treated with gold(III) porphyrin Ia. SK-N-SH and SK-N-AS were treated with gold(III) porphyrin Ia (0.5 $\mu \mathrm{M})$ for $8 \mathrm{~h}$ and Smac and cytochrome c in cytosol were detected by western blot analysis. SK-N-SH and SK-N-AS cells were also treated with gold (III) porphyrin Ia (0.5 $\mu \mathrm{M})$ for different periods of time. Western blotting was applied to analyse the expression of full-length XIAP (53 kDa) (B), full-length (47 kDa) and cleaved caspase-9 (37/35 kDa) (C), fulllength $(35 \mathrm{kDa})$ and cleaved caspase-3 $(19 / 17 \mathrm{kDa})(\mathbf{D})$, and full-length $(1 / 6 \mathrm{kDa})$ and cleaved PARP $(89 \mathrm{kDa})(\mathbf{E})$.

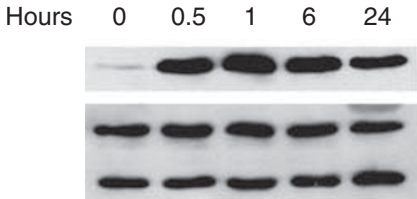

SK-N-SH

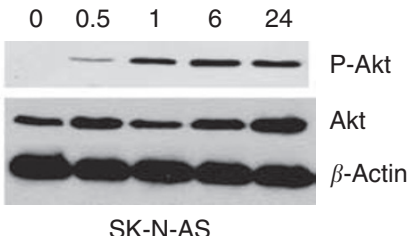

SK-N-AS
Figure 4 Effect of gold(III) porphyrin la on induction of Akt activity. SK-N-SH and SK-N-AS cells were treated with gold(III) porphyrin la $(0.5 \mu \mathrm{M})$ for different periods of time. Akt phosphorylation and total Akt expression was analysed by western blotting.

\section{DISCUSSION}

Gold(III) porphyrin 1a, a chemically synthetic compound with a simple chemical structure, is a novel class of antitumour drug (Che et al, 2003). It displayed antiproliferative activity in several types of human cancer cells (Wang et al, 2005, 2006; Lum et al, 2006). However, the precise target and mechanism of action of gold(III) porphyrin 1a on tumour cells remain largely unknown.

In this study, we showed that gold(III) porphyrin 1a induced apoptosis and growth inhibition in neuroblastoma cells, including MDR cells in vivo and in vitro. Importantly, there is no cytotoxicity in normal liver cells and peripheral blood mononuclear cells (data not shown). This in vitro result was confirmed by that of the in vivo study. Gold(III) porphyrin 1a treatment was active against neuroblastoma xenografts and significantly inhibited tumour growth. These data show that the antitumor activity exerted by gold(III) porphyrin 1a is effective, in vivo, in treating cancer.

Numerous stimuli and stresses are able to stimulate the release of apoptogenic factors from the mitochondrial intermembrane space, leading to the programmed demise of the cell. To understand the mechanism by which gold(III) porphyrin 1a induces apoptosis of tumour cells, we have examined the changes in the expression and localisation of several apoptosis-related proteins in neuroblastoma cells exposed to gold(III) porphyrin 1a. The western blot analysis revealed that gold(III) porphyrin 1a induced the release of cytochrome $c$ and Smac/Diablo, activation of caspase- 9 and -3 , and PARP cleavage, indicating that gold(III) porphyrin 1a-induced apoptosis might be primarily mediated by the mitochondrial (intrinsic) apoptosis pathway. We further explored the expression of XIAP in neuroblastoma cell lines. We found that SK-N-SH and SK-N-AS expressed high levels of XIAP. These findings correlated with previous studies showing that IAPs are highly expressed in many tumours and contribute to the resistance of cancers to apoptosis (Deveraux et al, 1998; Salvesen and Duckett, 2002; Kamsteeg et al, 2003). Treatment of the neuroblastoma cells with gold(III) porphyrin la inhibited the expression of XIAP. It has been shown that the cleavage of XIAP may be one of the mechanisms by which cell death programs circumvent the antiapoptotic barrier posed by XIAP (Deveraux 

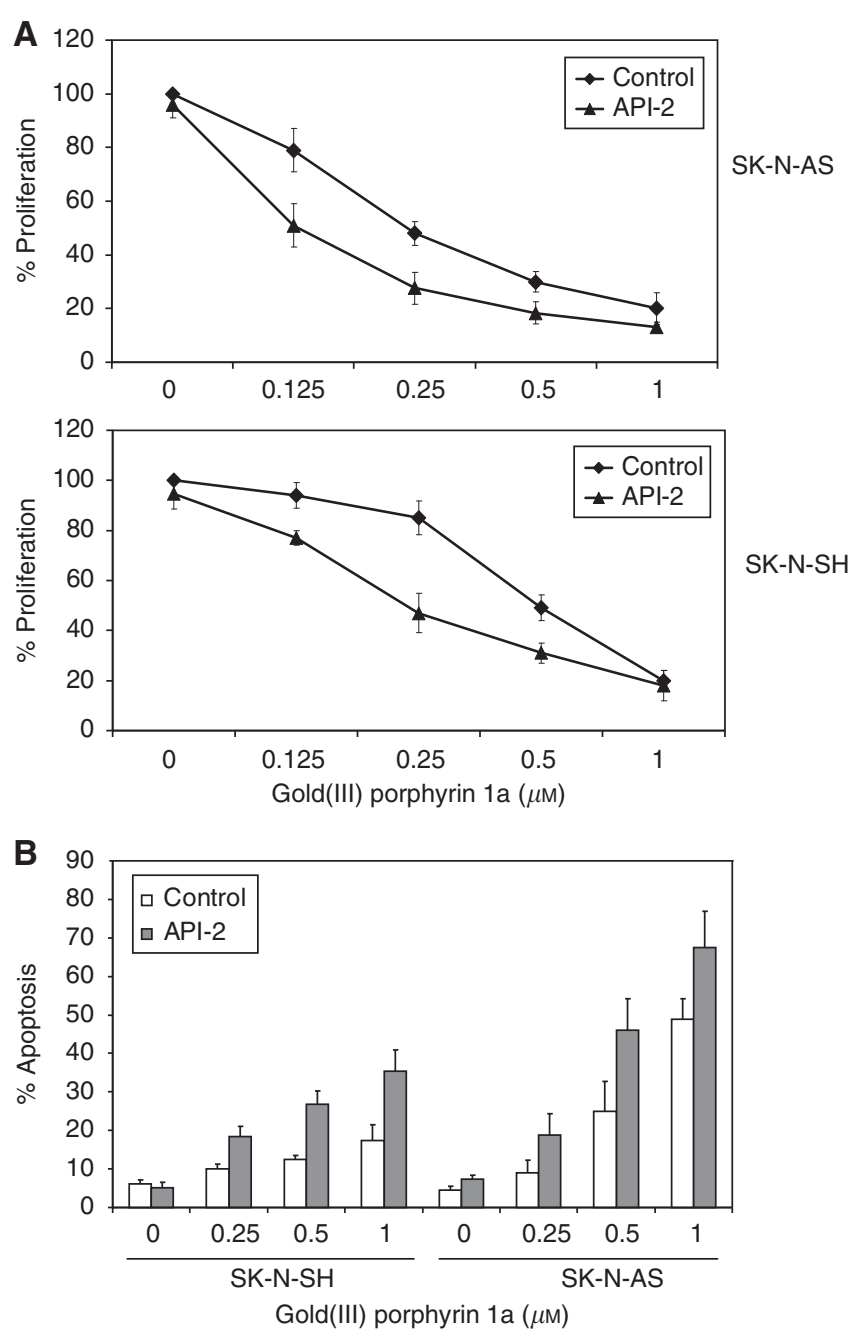

Figure 5 Inhibition of Akt activity sensitises cells to gold(III) porphyrin Ia-induced proliferative inhibition and apoptosis. (A) SK-N-SH and SK-NAS cells were pretreated with or without API-2 (I $\mu \mathrm{M})$ for $2 \mathrm{~h}$ and then cultured with gold(III) porphyrin la for $48 \mathrm{~h}$. Proliferation was assessed by MTT assay. Each treatment group contained eight replicates. Data were expressed as mean \pm s.d.; similar results were obtained from repeated experiments. (B) SK-N-SH and SK-N-AS cells were pretreated with or without API-2 (I $\mu \mathrm{M})$ for $2 \mathrm{~h}$ and then cultured with gold(III) porphyrin la for $24 \mathrm{~h}$. Apoptosis was assessed by Annexin $\mathrm{V}$ binding assay. Data were expressed as mean \pm s.d.; similar results were obtained from duplicate experiments.

et al, 1999). This may explain our findings of caspase-9 activation after gold(III) porphyrin 1a treatment.

Recent study showed that the majority of cytochrome $c$ release was abolished in the Smac-KO cells, suggesting that Smac plays an important role in promoting cytochrome $c$ release and full execution of NSAID-induced apoptosis (Bank et al, 2008). Studies by other groups also showed that the release of different mitochondrial apoptogenic proteins is coordinated and occurs through different time courses (Munoz-Pinedo et al, 2006). Our data showed for the first time that gold(III) porphyrin 1a not only enhanced cytochrome $c$ release but also promoted Smac release. Smac release may be critical for its ability to promote caspase activation and cytochrome $c$ release during gold(III) porphyrin 1ainduced apoptosis. The release of Smac and cytochrome $c$ may have synergetic role on apoptosis by the activation of caspase- 9 and inhibition of XIAP. Further study is needed to explore the requirement of Smac for full execution of gold(III) porphyrin 1ainduced apoptosis in neuroblastoma cells.
It is well documented that Akt can promote cellular survival. Akt is activated in response to many different growth factors (Datta et al, 1999). Previous studies also showed that Akt plays important roles in cell survival when cells are treated with different apoptotic stimuli such as growth factor withdrawal, UV irradiation, and etoposide (Widmann et al, 1998). Akt protects cells from apoptosis by phosphorylating and inactivating several key apoptotic molecules: Bad, procaspase-9, and FKHR1 (Pommier et al, 2004). Our results showed for the first time that gold(III) porphyrin 1a not only enhanced neuroblastoma apoptosis but also activated Akt, the survival signal. Inhibition of Akt activation sensitised neuroblastoma cells to the effects of antiproliferation and apoptosis by gold(III) porphyrin 1a. The antiproliferation and apoptosis induced by gold(III) porphyrin 1a on cells could be enhanced by the inhibition of Akt activation pharmacologically with specific Akt inhibitor, suggesting that Akt activation might play a role in the protection against the effects of apoptosis and growth inhibition of gold(III) porphyrin 1a in neuroblastoma. To our knowledge, these studies are the first to show that Akt is a survival factor for neuroblastoma cells under conditions of with administration of gold(III) porphyrin 1a, which are similar to the previous studies that showed that the Akt activity was induced by chemotherapy, doxorubicin, trastuzumab, or tamoxifen (Clark et al, 2002). This phenomenon was also observed by Tang et al (2001), who showed that Akt was activated in response to apoptotic stimuli: staurosporine and etoposide.

What is the mechanistic connection between gold(III) porphyrin 1a and Akt induction? It has been shown that gold(III) porphyrin 1a can generate ROS (Wang et al, 2005) which may contribute to Akt activation in a similar way to hydrogen peroxide as some apoptotic stimuli such as doxorubicin-generated ROS and UV B irradiation-induced DNA damage can activate Akt (Ushio-Fukai et al, 1999; Van der Kaay et al, 1999; Wang et al, 2000). However, the activation of Akt by hydrogen peroxide occurred within minutes and was transient, whereas our study showed that gold(III) porphyrin 1a-induced activation of Akt can last for at least $24 \mathrm{~h}$ and was not transient. This result suggests that other mechanisms may be involved to alter Akt levels in neuroblastoma cells in response to gold(III) porphyrin 1a. Other reports have showed that Akt can also be activated by other factors such as heat-shock proteins (Konishi et al, 1997; Rane et al, 2003; Solit et al, 2003) or by other cellular stress such as hypoxia (AlvarezTejado et al, 2001). Therefore, further studies are needed to identify the mechanisms of Akt activation induced by gold(III) porphyrin 1a.

Akt activation contributes to chemotherapeutic resistance and radiation resistance in breast, lung, prostate and ovarian carcinomas, leukaemia and melanoma (Krasilnikov et al, 1999; Wellbrock et al, 1999; Brognard et al, 2001; Chen et al, 2001; Stassi et al, 2005). It has been reported that a growth/survival factor-stimulated mechanism leading to chemoresistance in neuroblastoma is mediated by the PI3K/Akt signalling pathway (Li and Thiele, 2007). These findings raise the possibility of a new therapeutic approach in neuroblastoma that would target Akt to enhance the efficacy of chemotherapeutics. Combination regimens of gold(III) porphyrin 1a with an Akt inhibitor might have even great efficacy in MDR neuroblastoma cells. Several small-molecule inhibitors of the PI3K/Akt pathway are in active preclinical development (Powis et al, 2006). Specific Akt inhibitors such as API-2, a cell-permeable tricyclic nucleoside that selectively inhibits cellular pAkt by targeting the Akt effector molecule, do so without inhibition of activation of the upstream PI3K and PDK1 or downstream molecules (Yang et al, 2004). Although neuroblastoma SK-N-SH cell line assessed was less sensitive to gold(III) porphyrin 1a compared with SK-N-AS in vitro and in vivo, inhibition of Akt activation enhanced gold(III) porphyrin la response in these cells. Thus, p-Akt and its upstream activating pathway, PI3K, may have a critical role in 
conferring resistance to apoptosis induction by gold(III) porphyrin 1a. As Akt is vital for endothelial cell function and vascular integrity, its inhibition might also potentiate the antitumour effects of gold(III) porphyrin 1a (Somanath et al, 2006).

Taken together, our data emphasise that gold(III) porphyrin 1a is a mitochondrial apoptotic stimulus and reveal the potential importance of Akt as a therapeutic target in neuroblastoma. Akt may be considered as a molecular 'brake' that neuroblastoma cells rely on to slow down gold(III) porphyrin 1a-induced apoptosis and antiproliferation. It is possible that the activation of Akt inhibits the desired antitumor effects of gold(III) porphyrin 1a, particularly in the cells in which Akt is largely amplified. Inhibition of Akt activity by specific inhibitor can remove the apoptotic 'brake' and sensitise the neuroblastoma cells to gold(III) porphyrin 1a treatment.

\section{ACKNOWLEDGEMENTS}

This study was supported by Hong Kong Research Grants Council (HKU 7250/02M) and National Science Foundation (DMS0714589).

\section{REFERENCES}

Alvarez-Tejado M, Naranjo-Suarez S, Jimenez C, Carrera AC, Landazuri MO, del Peso L (2001) Hypoxia induces the activation of the phosphatidylinositol 3-kinase/Akt cell survival pathway in PC12 cells: protective role in apoptosis. $J$ Biol Chem 276: $22368-22374$

Bank A, Wang P, Du C, Yu J, Zhang L (2008) SMAC mimetics sensitize nonsteroidal anti-inflammatory drug-induced apoptosis by promoting caspase-3-mediated cytochrome $c$ release. Cancer Res 68: 276-284

Bossy-Wetzel E, Newmeyer DD, Green DR (1998) Mitochondrial cytochrome $\mathrm{c}$ release in apoptosis occurs upstream of DEVD-specific caspase activation and independently of mitochondrial transmembrane depolarization. ЕМBO J 17: $37-49$

Brognard J, Clark AS, Ni Y, Dennis PA (2001) Akt/protein kinase B is constitutively active in non-small cell lung cancer cells and promotes cellular survival and resistance to chemotherapy and radiation. Cancer Res 61: 3986-3997

Che CM, Sun RW, Yu WY, Ko CB, Zhu N, Sun H (2003) Gold(III) porphyrins as a new class of anticancer drugs: cytotoxicity, DNA binding and induction of apoptosis in human cervix epitheloid cancer cells. Chem Commun (Camb) 1718-1719

Chen X, Thakkar H, Tyan F, Gim S, Robinson H, Lee C, Pandey SK, Nwokorie C, Onwudiwe N, Srivastava RK (2001) Constitutively active Akt is an important regulator of TRAIL sensitivity in prostate cancer. Oncogene 20: 6073-6083

Cinatl Jr J, Cinatl J, Kotchetkov R, Vogel JU, Woodcock BG, Matousek J, Pouckova P, Kornhuber B (1999) Bovine seminal ribonuclease selectively kills human multidrug-resistant neuroblastoma cells via induction of apoptosis. Int J Oncol 15: $1001-1009$

Clark AS, West K, Streicher S, Dennis PA (2002) Constitutive and inducible Akt activity promotes resistance to chemotherapy, trastuzumab, or tamoxifen in breast cancer cells. Mol Cancer Ther 1: 707-717

Crow MT, Mani K, Nam YJ, Kitsis RN (2004) The mitochondrial death pathway and cardiac myocyte apoptosis. Circ Res 95: 957-970

Danial NN, Korsmeyer SJ (2004) Cell death: critical control points. Cell 116: $205-219$

Datta SR, Brunet A, Greenberg ME (1999) Cellular survival: a play in three Akts. Genes Dev 13: 2905-2927

Deveraux QL, Leo E, Stennicke HR, Welsh K, Salvesen GS, Reed JC (1999) Cleavage of human inhibitor of apoptosis protein XIAP results in fragments with distinct specificities for caspases. $E M B O \mathrm{~J}$ 18: $5242-5251$

Deveraux QL, Roy N, Stennicke HR, Van Arsdale T, Zhou Q, Srinivasula SM, Alnemri ES, Salvesen GS, Reed JC (1998) IAPs block apoptotic events induced by caspase- 8 and cytochrome $c$ by direct inhibition of distinct caspases. EMBO J 17: 2215-2223

Du C, Fang M, Li Y, Li L, Wang X (2000) Smac, a mitochondrial protein that promotes cytochrome $c$-dependent caspase activation by eliminating IAP inhibition. Cell 102: $33-42$

Jetzt A, Howe JA, Horn MT, Maxwell E, Yin Z, Johnson D, Kumar CC (2003) Adenoviral-mediated expression of a kinase-dead mutant of Akt induces apoptosis selectively in tumor cells and suppresses tumor growth in mice. Cancer Res 63: 6697-6706

Kamsteeg M, Rutherford T, Sapi E, Hanczaruk B, Shahabi S, Flick M, Brown D, Mor G (2003) Phenoxodiol - an isoflavone analog - induces apoptosis in chemoresistant ovarian cancer cells. Oncogene 22: $2611-2620$
Konishi H, Matsuzaki H, Tanaka M, Takemura Y, Kuroda S, Ono Y, Kikkawa U (1997) Activation of protein kinase B (Akt/RAC-protein kinase) by cellular stress and its association with heat shock protein Hsp27. FEBS Lett 410: 493-498

Krasilnikov M, Adler V, Fuchs SY, Dong Z, Haimovitz-Friedman A, Herlyn M, Ronai Z (1999) Contribution of phosphatidylinositol 3-kinase to radiation resistance in human melanoma cells. Mol Carcinog 24: 64-69

Li Z, Thiele CJ (2007) Targeting Akt to increase the sensitivity of neuroblastoma to chemotherapy: lessons learned from the brain-derived neurotrophic factor/TrkB signal transduction pathway. Expert Opin Ther Targets 11: $1611-1621$

Lum CT, Yang ZF, Li HY, Wai-Yin Sun R, Fan ST, Poon RT, Lin MC, Che CM, Kung HF (2006) Gold(III) compound is a novel chemocytotoxic agent for hepatocellular carcinoma. Int $J$ Cancer 118: $1527-1538$

Maris JM, Matthay KK (1999) Molecular biology of neuroblastoma. J Clin Oncol 17: 2264-2279

Matthay KK, Villablanca JG, Seeger RC, Stram DO, Harris RE, Ramsay NK, Swift P, Shimada H, Black CT, Brodeur GM, Gerbing RB, Reynolds CP (1999) Treatment of high-risk neuroblastoma with intensive chemotherapy, radiotherapy, autologous bone marrow transplantation, and 13-cis-retinoic acid. Children's Cancer Group. $N$ Engl J Med 341: $1165-1173$

Munoz-Pinedo C, Guio-Carrion A, Goldstein JC, Fitzgerald P, Newmeyer DD, Green DR (2006) Different mitochondrial intermembrane space proteins are released during apoptosis in a manner that is coordinately initiated but can vary in duration. Proc Natl Acad Sci USA 103: $11573-11578$

Nicholson KM, Anderson NG (2002) The protein kinase B/Akt signalling pathway in human malignancy. Cell Signal 14: 381-395

Osaki M, Oshimura M, Ito H (2004) PI3K-Akt pathway: its functions and alterations in human cancer. Apoptosis 9: 667-676

Pommier Y, Sordet O, Antony S, Hayward RL, Kohn KW (2004) Apoptosis defects and chemotherapy resistance: molecular interaction maps and networks. Oncogene 23: 2934-2949

Potts PR, Singh S, Knezek M, Thompson CB, Deshmukh M (2003) Critical function of endogenous XIAP in regulating caspase activation during sympathetic neuronal apoptosis. J Cell Biol 163: 789-799

Powis G, Ihle N, Kirkpatrick DL (2006) Practicalities of drugging the phosphatidylinositol-3-kinase/Akt cell survival signaling pathway. Clin Cancer Res 12: 2964-2966

Rane MJ, Pan Y, Singh S, Powell DW, Wu R, Cummins T, Chen Q, McLeish KR, Klein JB (2003) Heat shock protein 27 controls apoptosis by regulating Akt activation. J Biol Chem 278: $27828-27835$

Salvesen GS, Duckett CS (2002) IAP proteins: blocking the road to death's door. Nat Rev Mol Cell Biol 3: 401-410

Solit DB, Basso AD, Olshen AB, Scher HI, Rosen N (2003) Inhibition of heat shock protein 90 function down-regulates Akt kinase and sensitizes tumors to Taxol. Cancer Res 63: 2139-2144

Somanath PR, Razorenova OV, Chen J, Byzova TV (2006) Akt1 in endothelial cell and angiogenesis. Cell Cycle 5: 512-518

Stassi G, Garofalo M, Zerilli M, Ricci-Vitiani L, Zanca C, Todaro M, Aragona F, Limite G, Petrella G, Condorelli G (2005) PED mediates AKTdependent chemoresistance in human breast cancer cells. Cancer Res 65: $6668-6675$ 
Tang D, Okada H, Ruland J, Liu L, Stambolic V, Mak TW, Ingram AJ (2001) Akt is activated in response to an apoptotic signal. J Biol Chem 276: $30461-30466$

Ushio-Fukai M, Alexander RW, Akers M, Yin Q, Fujio Y, Walsh K, Griendling KK (1999) Reactive oxygen species mediate the activation of Akt/protein kinase B by angiotensin II in vascular smooth muscle cells. J Biol Chem 274: 22699-22704

Van der Kaay J, Beck M, Gray A, Downes CP (1999) Distinct phosphatidylinositol 3-kinase lipid products accumulate upon oxidative and osmotic stress and lead to different cellular responses. J Biol Chem 274: $35963-35968$

Wang X, McCullough KD, Franke TF, Holbrook NJ (2000) Epidermal growth factor receptor-dependent Akt activation by oxidative stress enhances cell survival. J Biol Chem 275: 14624-14631

Wang Y, He QY, Che CM, Chiu JF (2006) Proteomic characterization of the cytotoxic mechanism of gold (III) porphyrin 1a, a potential anticancer drug. Proteomics 6: $131-142$
Wang Y, He QY, Sun RW, Che CM, Chiu JF (2005) GoldIII porphyrin 1a induced apoptosis by mitochondrial death pathways related to reactive oxygen species. Cancer Res 65: 11553-11564

Weinstein JL, Katzenstein HM, Cohn SL (2003) Advances in the diagnosis and treatment of neuroblastoma. Oncologist 8: 278-292

Wellbrock C, Fischer P, Schartl M (1999) PI3-kinase is involved in mitogenic signaling by the oncogenic receptor tyrosine kinase Xiphophorus melanoma receptor kinase in fish melanoma. Exp Cell Res 251: $340-349$

Widmann C, Gibson S, Johnson GL (1998) Caspase-dependent cleavage of signaling proteins during apoptosis. A turn-off mechanism for antiapoptotic signals. J Biol Chem 273: $7141-7147$

Yang L, Dan HC, Sun M, Liu Q, Sun XM, Feldman RI, Hamilton AD, Polokoff M, Nicosia SV, Herlyn M, Sebti SM, Cheng JQ (2004) Akt/ protein kinase B signaling inhibitor-2, a selective small molecule inhibitor of Akt signaling with antitumor activity in cancer cells overexpressing Akt. Cancer Res 64: 4394-4399 